\title{
Slope stability analysis using recent metaheuristic techniques: a comprehensive survey
}

\author{
Mayank Mishra ${ }^{1}$. Venkata Ramana Gunturi² ${ }^{2}$ Tiago Filipe Da Silva Miranda ${ }^{3}$
}

Received: 6 September 2019 / Accepted: 15 November 2019 / Published online: 25 November 2019

(c) Springer Nature Switzerland AG 2019

\begin{abstract}
In the framework of designing civil engineering structures such as dams and road embankments, slope stability assessment is essential. Many slope stability assessment methods based on swarm intelligence algorithms and artificial intelligence techniques have been developed in the past decade. Therefore, this paper aims to provide an up-to-date overview of slope stability by summarizing the review of applications of several metaheuristics in this field with their advantages and limitations. In this study, we present recent swarm intelligence methods and machine learning techniques used for assessing the stability of slopes and compare them with the antlion optimiser (ALO) technique. The factor of safety related to every slip surface searched was computed using Morgenstern-Price method. The performance of the proposed ALO is evaluated and validated for four slope examples against recent metaheuristics. Finally, the work carried out will help practitioners as they can now have all swarm intelligence approaches combined in one paper and will help in choosing them the right technique based on their application.
\end{abstract}

Keywords Factor of safety · Metaheuristic algorithms · Artificial intelligence · Optimisation techniques · Limit equilibrium method

\section{Introduction}

Slope stability assessment is a crucial step in civil engineering for designing earth dams and road embankments. The computation of the minimum factor of safety (FS) is paramount to slope assessments because misjudgements may cause catastrophic slope failures and loss of life as reported in previous studies [1-4]. A FS higher than 1 means that the slope is stable but normally in engineering applications a minimum value of 1.5 is required for static gravity loading and between 1.2-1.3 for seismic conditions particularly when slope failure causes major damages such as slopes beneath bridge abutments, major roadways and retaining structures. Generally, for slope stability assessment several trial slip surfaces are considered to determine the one with the minimum FS value. The identification of the critical slip surface is the primary step, which is regularly performed using traditional limit equilibrium methods (LEM) in professional practice. Recently, bio-inspired optimisation techniques have made assessing slope stability easier, particularly for heterogeneous slopes and slopes with a weak band of a soil layer sandwiched between two strong layers [5]. In cases involving the aforementioned slope conditions, the problem to be analysed is nonlinear with a noncircular slip surface, whereas the search requires more than three variables. The three variables for the circular slip surface are coordinates of the center of the slip surface (horizontal, vertical) and radius of the slip surface.

Several techniques, including prominent traditional techniques to recent optimisation techniques, used to

Mayank Mishra, mayank@iitbbs.ac.in; mayank_mishra@outlook.in; Venkata Ramana Gunturi, ramana@civil.iitd.ac.in; Tiago Filipe Da Silva Miranda, tmiranda@civil.uminho.pt | ${ }^{1}$ School of Infrastructure, Indian Institute of Technology, Bhubaneswar, Odisha 752050, India. ${ }^{2}$ Department of Civil Engineering, Indian Institute of Technology, Delhi 110016, India. ${ }^{3}$ ISISE, Institute of Science and Innovation for Bio-Sustainability (IB-S), Department of Civil Engineering, University of Minho, 4800-058 Guimarães, Portugal. 
assess slope stability are summarised in this paper. The most prevalently used method for performing slope stability calculations is traditional limit equilibrium method [6-11]. Classical techniques for assessing slope stability are unsuitable for several cases such as slopes with non-circular slip surfaces having multiple local minima solution and require a close by preliminary solution to obtain a valid result. A traditional LEM can identify the critical slip surface; however, the method fails to identify a global minimum in certain situations causing premature convergence at a local minimum [12]. Furthermore, other techniques, including finite difference [13] and finite element strength reduction techniques [14-17] are extensively used. By contrast, finite element techniques need not assume the pattern and position of trial slip surfaces and introduce other complexities, such as defining stress-strain relations of soils. Monte Carlo methods used for slope stability calculation $[18,19]$ should have quality inputs to arrive to a desirable FS and they often underestimate the probability of slope failures. Furthermore, MC methods are slow in computation as large sample size is required to obtain desirable result.

At present, intelligent algorithms such as harmony search (HS) [20], ant colony optimisation (ACO) [21-23], particle swarm optimisation (PSO) [24, 25], simulated annealing (SA) [26], artificial bee colony (ABC) [27], cuckoo search (CS) [28], firefly algorithm (FA) [28], fish swarm optimisation (FSO) [29], gravitational search algorithm (GSA) [30-32], big bang - big crunch (BB-BC) optimisation [33], relevance vector machine [34], mutative scaled chaos (MSC) [35], tabu search (TS) [36], genetic algorithms (GA) [37-42], fireworks algorithm (FWA) [43], black hole algorithm (BHA) [44], immunised evolutionary programming (IEP) [45], differential evolution (DE) [46, 47], evolutionary strategy (ES) [46, 47], and biogeography-based optimisation (BBO) $[46,47]$, imperialistic competitive algorithm (ICA) [48], multiverse optimisation algorithm (MVO) [49] and teaching-learning-based optimisation (TLBO) [50] have been applied for slope stability analysis. Moreover, the hybridisation of these algorithms, such as CS with boundary constraint (CS-EB) [51], PSO with HS (PSO-HS) [52], ACO with simulated annealing (ACO-SA) [53], and GSA with sequential quadratic programming (GSA-SQP) [54] are used. Furthermore, artificial neural networks (ANNs) [55-64], reliability index [65] and fuzzy logic [66] have also been used to analyse the stability of slopes and earth dams. These studies indicate the potential of metaheuristic algorithms for assessing slope stability.

The FS results obtained using these metaheuristic approaches get trapped in local optimum because of the selection of an inaccurate set of parameters. For e.g., ACO has limitations because it may converge to local optimum providing inaccurate results, which is attributed to the selection of inaccurate tuning parameters, and requires high computation time. A similar case used GA for optimisation because it required the careful selection of crossover and mutation rates. Additionally, PSO and $A B C$ use inertia weights, social and cognitive parameters, and the number of bees and limits, respectively. Hybrid approaches require complex actions, such as hybridising, which are difficult to include for practitioners. In addition, the ANN has severe limitations because it requires a large database comprising several slope failure conditions and can only be applied to the considered case. Artificial intelligence (Al) techniques [67] utilised for accurate prediction of slope stability require hyperparameter and weight tuning. Table 1 summarises different algorithmic specific parameters and their numerical values tuned for computing FS of slopes. Thus, all optimisation techniques excluding BHA and TLBO, require careful parameter tuning to capture global optima. Hence, different metaheuristic approaches that do not encounter the aforementioned limitation of parameter tuning are required, which can evade this local minima in most cases.

The no-free-lunch theorem [68] entices scientists to continually come up with novel metaheuristic algorithms and employ them to their respective fields of interest such as optimizing the mechanical stabilization of earth walls [69] and reinforced concrete cantilever retaining walls [70]. In 2015, Mirjalili [71] proposed a new optimisation method termed ALO whose description is provided in Sect. 2. Several studies $[72,73]$ have compared ALO with GA and PSO and indicated the superior performance of ALO in solving non-convex and complex optimisation problems. The ALO algorithm has been successfully applied to solve numerous engineering problems [72-79]. In this particular application, a procedure for employing an ALO method was developed and adapted for calculating the minimum FS of a slope, and four benchmark slopes were considered. A comparison between different results obtained using other state-ofthe-art approaches and the current technique indicates advantages and limitations of the new method. Different algorithms may have different fitness function evaluations consumed per iteration $[80,81]$, and therefore, the comparison of FS with other metaheuristics approaches is considered.

The paper is organised as follows: Sect. 1 introduces the previous studies performing geotechnical slope stability calculations by using intelligent algorithms and limitations of traditional techniques; Sect. 2 introduces the ALO algorithm; Sect. 3 discusses slope stability calculations of the ALO algorithm; in Sect. 4, four case studies are presented to validate the technique; and finally, in Sect. 5 conclusions are presented. 
Table 1 Literature review on algorithmic specific parameters used in ALO and other metaheuristic approches for slope stability analyses

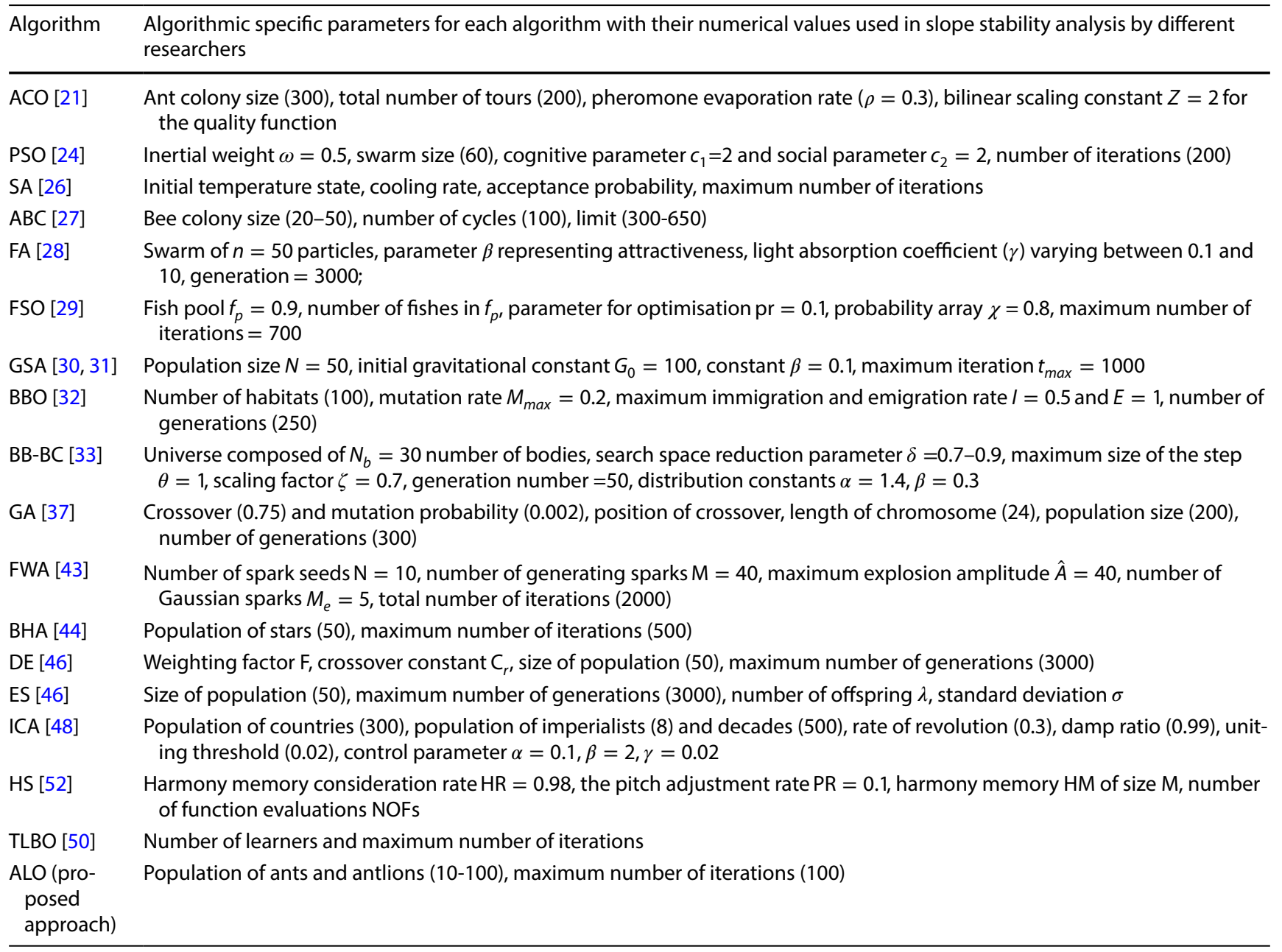

Acronyms of all algorithms used are reported in Sect. 1

\section{Overview of ALO algorithm}

ALO is a bio-inspired optimisation algorithm popularised by Mirjalili [71]. It is a swarm-based algorithm, which employs random walks and roulette wheel selections biased using fitness functions for selecting control variables, and therefore, probability of falling for local minima is considerably low. The five primary steps involved in ALO are as follows: First, an antlion creates a cone-shaped sandpit (Fig. 1a). The antlion then hides from the ant (Fig. 1b, c) and waits for the ant to get trapped. When the ant enters cone-shaped sandpit, the antlion tosses sand outside the pit, compelling the ant
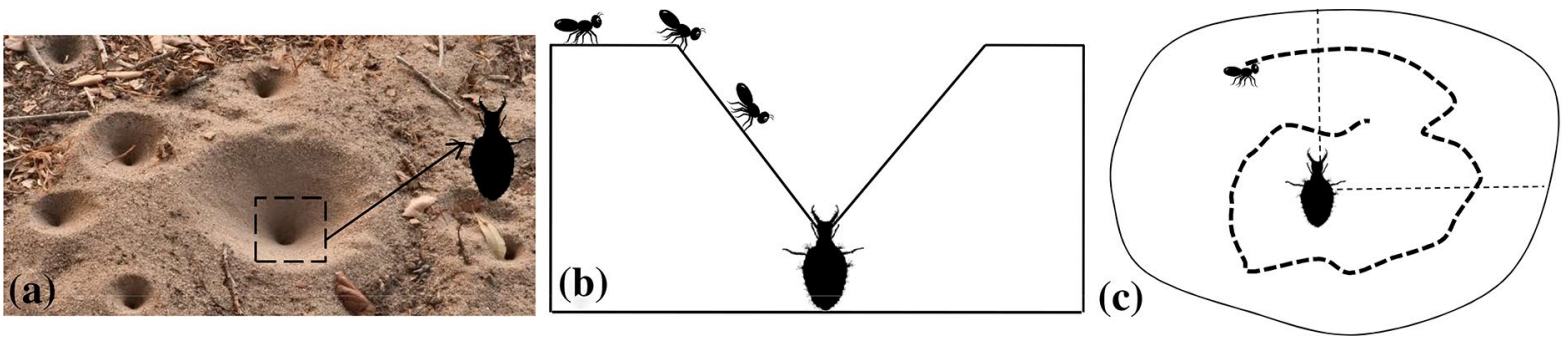

(d)

Fig. 1 a Cone-shaped trap of antlion with antlion hiding beneath the pit [78], $\mathbf{b}$ ants entering the trap [78], c random walk of ant in antlion's pit, and $\mathbf{d}$ ant being consumed by antlion 
to fall towards the edge of the pit. The ant is then pulled into the sandpit and consumed (Fig. 1d). Finally, the antlion throws the uneaten ant and plans for the next plot. The mathematical model of ALO is briefly explained as follows $[71,76,78]$ :

\subsection{Initialisation}

The ALO algorithm models the interaction of two populations, namely ants and antlions. The population of ants is equal to number of antlions, denoted by constant NP. The position of the ants (Eq. 1) is stored in matrix Mat ${ }_{\text {Ant }}$ where each row represents the values of control variables for a particular solution.

$\operatorname{Mat}_{\text {Ant }}=\left(\begin{array}{cccc}1 & 2 & \multicolumn{2}{c}{\text { Dim }} \\ \text { Ant }_{1,1} & \text { Ant }_{1,2} & \ldots & \text { Ant }_{1, \text { Dim }} \\ \text { Ant }_{2,1} & A n t_{2,2} & \ldots & \text { Ant }_{2, \text { Dim }} \\ \vdots & \vdots & \vdots & \vdots \\ \text { Ant }_{N P, 1} & \text { Ant }_{N P, 2} & \ldots & \text { Ant }_{N P, \text { Dim }}\end{array}\right){ }_{N P}$

where $A n t_{i, j}$ and Dim indicate the position of the $j$ th control variable for the ith ant and the total number of control variables, respectively. Mat $_{\text {OAnt }}$ (Eq. 2) and Fit are the matrix storing the fitness function associated with each ant position and the fitness function, respectively.

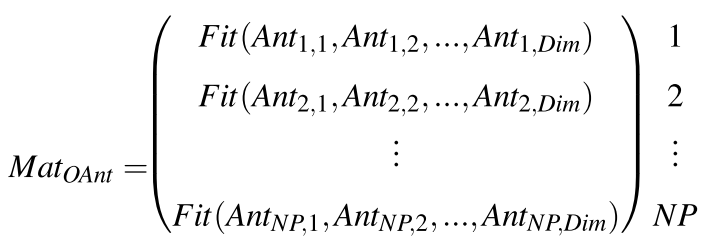

Moreover besides the ants, the antlions also hide whose matrices $\mathrm{Mat}_{\text {Antlion }}$ and $\mathrm{Mat}_{\text {OAntlion }}$ are used to store their scavenging positions and fitness values. After the initialisation step, the optimal antlion (antlion with the maximum fitness value) is selected as an elite.

\subsection{Digging traps}

The ALO algorithm utilises a roulette wheel mechanism to select antlions based on their fitness values. This process ensures high probability for ensnare ants for antlions with high fitness. For each ant, one antlion is selected, thus only one ant is trapped by one antlion.

\subsection{Sliding ants towards antlion}

Antlions start tossing sand away from the pit after realising that the ant is entering the trap. Thus boundaries of ants random walk (Fig. 1c) are updated as below:

$$
\begin{aligned}
& c_{i}(\text { Iter })=\frac{c_{i}(\text { Iter })}{10^{\omega} \frac{\text { Iter }}{\text { Iter }_{\max }}} \\
& d_{i}(\text { Iter })=\frac{\left.d_{i} \text { (Iter }\right)}{10^{\omega} \frac{\text { Iter }}{\text { Iter } \max }}
\end{aligned}
$$

where $c_{i}($ Iter $)$ and $d_{i}$ (Iter) are the vector containing minimum and maximum limits of the ith control variable, and Iter denotes the present iteration number and $\omega$, a constant based on range of ratio of Iter and $/$ ter $_{\max }$ (maximum number of iterations) [71] .

\subsection{Entrapment in antlion pits}

The subsequent equations below model the entrapment of ants in antlion pits, thus influencing their random walks:

$c_{i}^{m}($ Iter $)=$ Antlion $_{j}($ Iter $)+c_{i}($ Iter $)$

$d_{i}^{m}($ Iter $)=$ Antlion $_{j}($ Iter $)+d_{i}($ Iter $)$

\subsection{Random walk of ants in antlion pit}

In this step, the population of ants (Fig. 1b) is initialised using a random walk by the following equation:

$$
\begin{aligned}
P(\text { Iter })= & \left(0, \operatorname{cums}\left(2 r w_{1}-1\right), \operatorname{cums}\left(2 r w_{2}-1\right), \ldots,\right. \\
& \left.\operatorname{cums}\left(2 r w_{\text {Iter }_{\text {max }}}-1\right)\right)
\end{aligned}
$$

where cums provides accumulative sum, and $r w_{j}$ comprises of the jth row of the vector, whose value is 1 for random number generated greater than 0.5 and 0 otherwise. The walks are normalised by the following equation to keep them within bounds.

$P_{i}($ Iter $)=\frac{\left(P_{i}(\text { Iter })-a_{i}\right)\left(d_{i}(\text { Iter })-c_{i}(\text { Iter })\right)}{\left(b_{i}-a_{i}\right)}+c_{i}($ Iter $)$

where $P_{i}($ Iter $)$ is the updated using normalised position of the $i$ th control variable at the Iter. $a_{i}$ and $b_{i}$ are the lower and upper limits of the ith control variable, while the indices for $c_{i}$ (Iter) and $d_{i}$ (Iter) denote the lower and upper boundaries for the current iteration Iter.

\subsection{Elitism}

Parameters of the ant are now calculated based on the current optimal antlion and global optimal antlion to enhance 


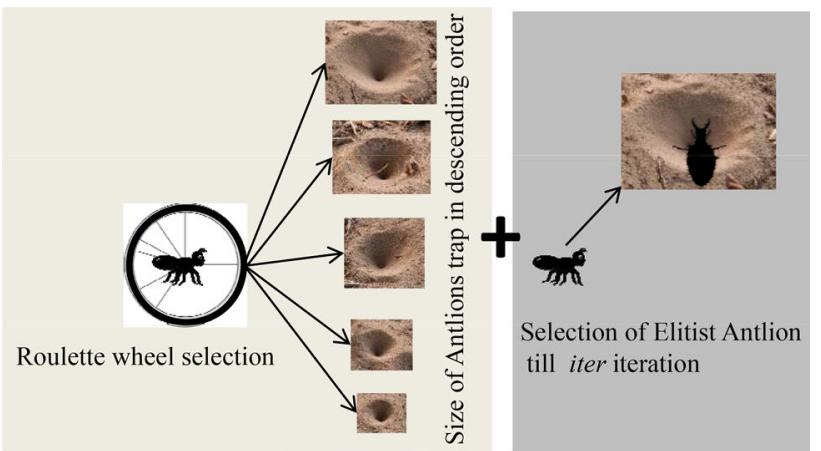

Fig. 2 Figure illustrating the update of ants position based on roulette wheel mechanism and elite antlion

the local and global search capability (Fig. 2). The position of the ith ant at current iteration is updated as follows:

$A n t_{i}($ Iter $)=\frac{R W_{\text {Roulette }}(\text { Iter })+R W_{\text {Elite }}(\text { Iter })}{2}$

where $R W_{\text {Roulette }}\left(\right.$ Iter) and $R W_{\text {Elite }}$ (Iter) are random walks of the $i$ th ant around antlion tabbed through roulette wheel mechanism and the elite antlion.

\subsection{Catching preys, rebuilding traps}

The fitness function for the position of the ant is calculated in this step. The fittest solution is replaced with the existing solution if it is superior $\left(\right.$ fit $\left(\right.$ Ant $_{i}($ Iter $\left.)\right)<$ fit $\left(\right.$ Antlion $_{j}($ Iter $\left.\left.)\right)\right)$ else the previous solution is retained as per the following equation:

Antlion $_{j}($ Iter $)=$ Ant $_{i}($ Iter $)$

The aforementioned steps in Sects. 2.2-2.7 are repeated until /ter $r_{\text {max }}$ are reached.

\section{Implementation of ALO to slope stability problem}

The method explained in Sect. 2 is modified to fit into a slope stability problem. The process comprises of the following steps: the generation of the trial slip surface, calculating the FS, and using ALO to identify the critical slip surface and compute its FS. A computer code is developed in MATLAB [82] to automatically search the critical slip surface using ALO.

\subsection{Generation of trial slip surface and FS computation}

Before calculating for the FS, the slip surface must be generated using a slip surface generation algorithm. Several such algorithms are available [20]. In this paper, the method proposed by Cheng [83] is used. The method by Cheng [83] is used as it was easy to implement in computer programming language and involved fewer ranges to be defined for control variables (only ranges of entry and exit points of slip surfaces). Figure 3 illustrates the method of generating the trial slip surface, where $y=G(x)$ and $B(x)$ represents the mathematical function of slope geometry and the equation of the bedrock, respectively.

In the first step, the failure mass is split into $N$ vertical segments having uniform width. Although from previous researches, horizontal slice methods developed by Lo and $\mathrm{Xu}$ [84] are having many advantages in solving for the safety factor of slopes, but for the scope of work only vertical slices are used in the present study. The failure surface is defined by $N+1$ vertices $\left[V_{1}, V_{2}, \ldots, V_{N+1}\right]$ as follows:

$V=\left[\left(x_{1}, y_{1}\right),\left(x_{2}, y_{2}\right), \ldots,\left(x_{N}, y_{N}\right),\left(x_{N+1}, y_{N+1}\right)\right]$

Values of $x_{1}$ (entry) and $x_{N+1}$ (exit) shown in Fig. 4a can be determined from engineering experience. Because all slices have the same width, $x$-coordinates of points from $x_{2}$ to $x_{N}$ (not control variables) can be determined by the following equation:

$x_{i+1}=x_{1}+\left(\frac{x_{N+1}-x_{1}}{N}\right) \times i$ for $i=1,2, \ldots, N-1, N$

For $y$ coordinates $y_{1}$ and $y_{N+1}$ can be computed using the geometry of the slope. To determine $y$ coordinates of other points, upper and lower bounds $\left(y_{\text {imax }}\right.$ and $\left.y_{i \min }\right)$ that represent slope geometry and bedrock profile, respectively, are considered. For optimisation algorithms, minimum and maximum limits of decision variables are pre-stated and constant during the optimisation. Lower and upper bounds of ordinates of vertices from $V_{2}$ till $V_{N}$ are set to $y_{i m i n}$ and $y_{\text {imax }}$ because their minimum and maximum value are dynamically defined using the following equation:

$y_{i}=y_{\text {imin }}+\left(y_{\text {imax }}-y_{i \min }\right) \times \sigma_{i} ; i=1, \ldots, N-1$

To summarise the optimisation process for identifying the critical slip surface having a minimum FS can be summarised as follows:

$$
\begin{aligned}
& \min f(x \leftarrow \mathbf{V}) \text { s.t } x_{l} \leq x_{1} \leq x_{u} ; x_{L} \leq x_{N+1} \leq x_{U} ; \\
& 0<\sigma_{i}<1 ; i=1, \ldots, N-1
\end{aligned}
$$

where $\mathbf{V}$ can be obtained using the aforementioned procedure. 


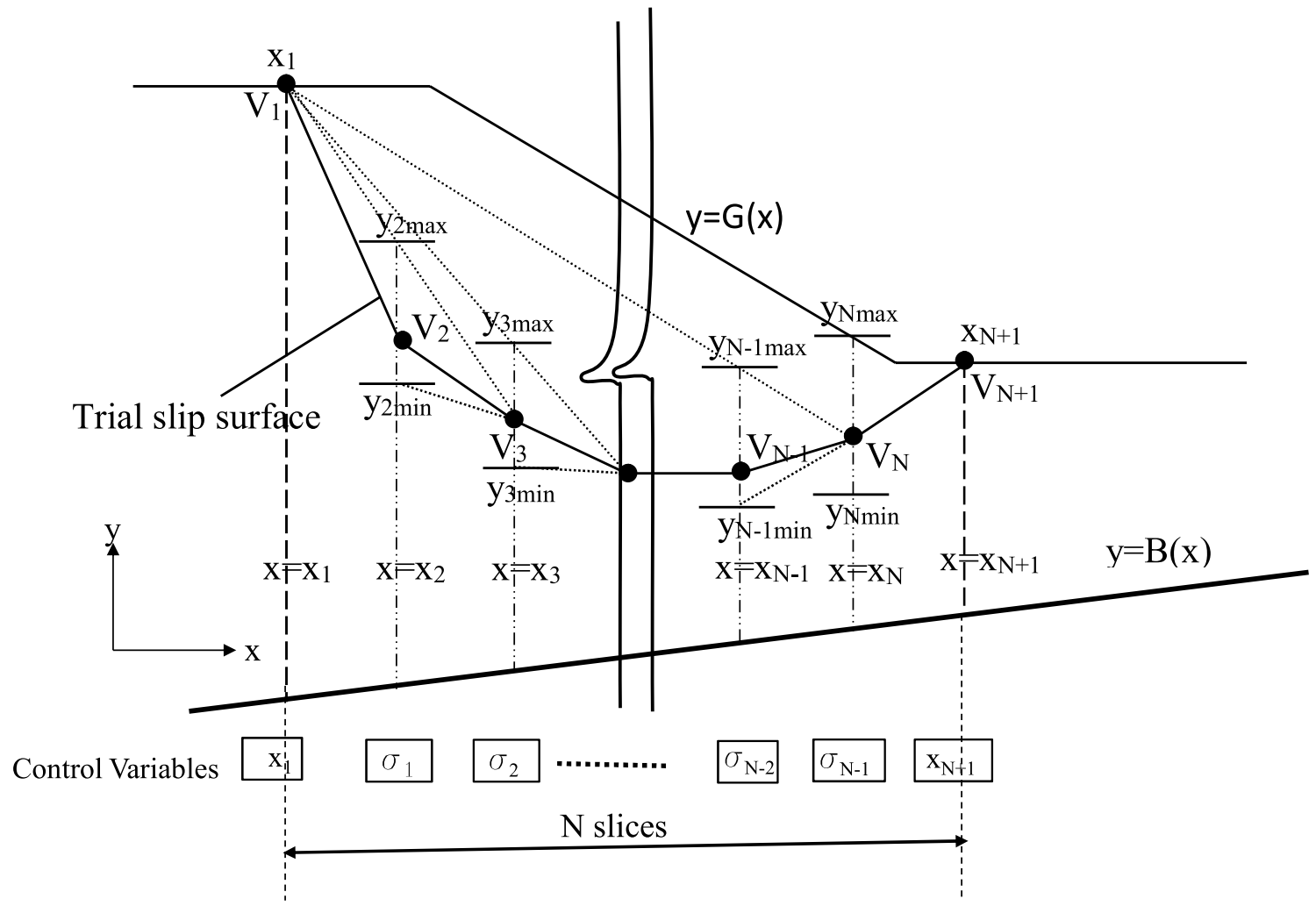

Fig. 3 Procedure for generating trial slip surfaces (modified from [83])

(a)

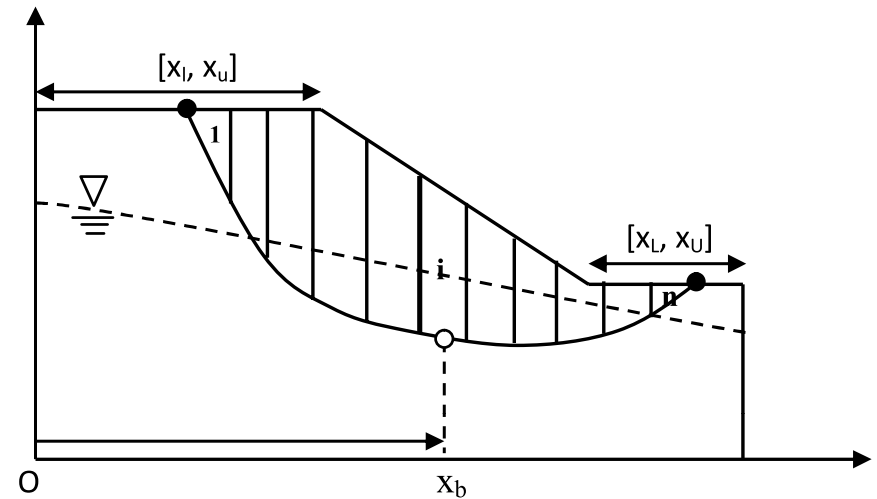

(b)

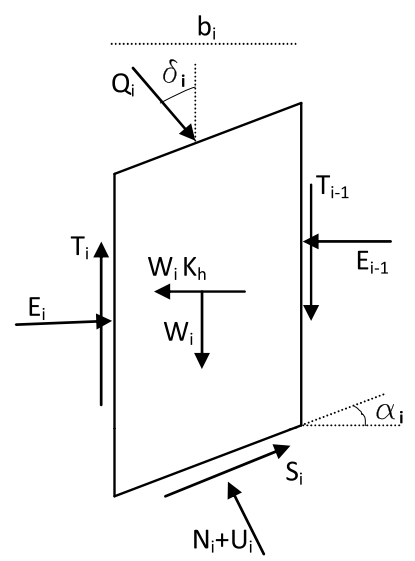

Fig. 4 a Sliding body showing division of slices and $\mathbf{b}$ forces acting on ith slice

For each trial slip surface generated by aforementioned procedure, Morgenstern-Price method [8] is used to compute the FS. However, equilibrium equations for force and moment summing over all slices are nonlinear and cannot be solved using a simple technique. In this paper, the nonlinear equations are solved using a numerical procedure developed by Zhu et al. [85] to obtain converging values for the FS and scaling factor $(\lambda)$. In Morgenstern-Price method, the ratio of interslice forces $\left(T_{i} / E_{i}\right)$ is assumed to be in a $\lambda f\left(x_{b}\right)$, and the relation can be expressed as follows:

$\frac{T_{i}}{E_{i}}=\lambda f\left(x_{b}\right)$

where $T_{i}$ and $E_{i}$ are shear interslice and normal interslice forces, respectively (Fig. 4b). $f\left(x_{b}\right)$ is the interslice force function that varies with respect to $x_{b}$ (Fig. 4a). 
The sliding mass is split into vertical slices and resolved into forces as shown in Fig. 4 a to evaluate the FS. From Fig. $4 \mathrm{~b}$, where $W_{i}=$ weight of $i$ th slice; $U_{i}=$ pore water pressure at the base of the ith slice; $N_{i}=$ effective normal force at the base of the ith slice; $Q_{i}=$ external surcharge load acting on the ith slice at inclination $\delta_{i}, \alpha_{i}$ $=$ horizontal angle of the slice base; $b_{i}=$ the width of the $i$ th slice; $K_{h}=$ horizontal seismic coefficient; and $E_{i}$ and $E_{i-1}$ are the normal interslice forces acting on left and right boundaries of the slice, respectively. For soil parameters, $c^{\prime}, \phi^{\prime}=$ effective cohesion and internal friction angle at the base of the ith slice. The following iterative algorithm is employed to evaluate the FS [85]:

i) Compute $R_{i}$ and $T_{i}$ for each slice by using the following equations:

$R_{i}=\left(W_{i} \cos \alpha_{i}-K_{h} W_{i} \sin \alpha_{i}-u_{i} b_{i} \sec \alpha_{i}\right) \tan \phi_{i}^{\prime}+c_{i} b_{i} \sec \alpha_{i}$

$T_{i}=W_{i} \sin \alpha_{i}+K_{h} W_{i} \cos \alpha_{i}$

ii) The initial trial values of the $F S$ and $\lambda$ must be selected through criteria [85], however $F S_{o}=1$ and $\lambda_{o}=0$ can be safely selected as an initial prediction.

iii) A constant interslice function is selected; that is, interslice forces are parallel, which is a particular case of Morgenstern-Price method, equivalent to Spencer method [86].

$f\left(x_{b}\right)=1$

iv) Compute coefficients $\Phi_{i}$ and $\psi_{i-1}$ for each slice by using the following equations:

$\Phi_{i}=\left(\sin \alpha_{i}-\lambda f_{i} \cos \alpha_{i}\right) \tan \phi_{i}^{\prime}+\left(\cos \alpha_{i}-\lambda f_{i} \sin \alpha_{i}\right) F S$

$\psi_{i-1}=\frac{\Phi_{i}}{\Phi_{i-1}}$

v) The $F S$ is calculated using Eq. 21 and $\Phi_{i}$ and $\psi_{i}$ are then determined using the current value of the $F S$. The $F S$ is recomputed using updated values of $\Phi_{i}$ and $\psi_{i}$.

$F S=\frac{\sum_{i=1}^{n-1}\left(R_{i} \prod_{j=1}^{n-1}\right)+R_{n}}{\sum_{i=1}^{n-1}\left(T_{i} \prod_{j=1}^{n-1}\right)+T_{n}}$

vi) Use Eqs. 22 and 23 to calculate $E_{i}$ and $\lambda$.

$E_{i}=\frac{\psi_{i-1} E_{i-1} \Phi_{i-1}+F S \times T_{i}-R_{i}}{\Phi_{i}}$

when $E_{o}$ and $E_{n}$ are the interslice force at boundaries and are set at 0 .

$$
\lambda=\frac{\sum_{i=1}^{n}\left[b_{i}\left(E_{i}+E_{i-1}\right) \tan \alpha_{i}+K_{h} W_{i} h_{i}\right]}{\sum_{i=1}^{n}\left[b_{i}\left(f_{i} E_{i}+f_{i-1} E_{i-1}\right)\right]}
$$

The steps (ii)-(vi) are reiterated until the values of the $F S$ and $\lambda$ are within set tolerance value. The aforementioned procedure was implemented in MATLAB and converged to the desired accuracy in less than 5 iterations.

\subsection{ALO application to slope stability problem}

In this application, the algorithm is modelled to determine the optimal FS for trial slip surfaces. The slope is divided into 30 slices having a total of 31 control variables. The variation in $x_{1}$ and $x_{31}$ is explained in Sect. 3.1, whereas all $\sigma_{1}-\sigma_{29}$ vary from $[0,1]$. The ALO algorithm is implemented in the following seven steps for slope stability problem:

Step 1 Generate the first array of population search agents and calculate their fitness. The values are defined for Dim + 1 variables (31 in this case) that represent coordinates of the trial slip surface as shown in Fig. 4a. The first step involves the initialisation of the algorithm by using a set of random ants roaming and antlions laying traps through the search space.

$$
\text { Mat }_{\text {Ant }}=\text { Mat }_{\text {Antlion }}=\left(\begin{array}{cccccc}
1 & 2 & 3 & & 30 & 31 \\
x_{1}^{1} & \sigma_{2}^{1} & \ldots & \ldots & \sigma_{29}^{1} & x_{31}^{1} \\
x_{1}^{2} & \sigma_{1}^{2} & \ldots & \ldots & \sigma_{29}^{2} & x_{31}^{2} \\
\vdots & \vdots & \vdots & \vdots & \vdots & \vdots \\
x_{1}^{N P} & \sigma_{1}^{N P} & \ldots & \ldots & \sigma_{29}^{N P} & x_{31}^{N P}
\end{array}\right)_{N P}
$$

Step 2 Sort antlions according to their fitness values in an ascending order, and select the antlion with the optimal fitness as the elite.

Step 3 Pick an antlion for each individual ant via roulette wheel. Antlions having a superior fitness value has higher probability of selection through this process.

3.1 Create a random walk (Eq. 7), and normalise it (Eq. 8). 3.2 Update the values of $c_{i}^{m}$ (Iter) and $d_{i}^{m}($ Iter) (Eqs. 5-6).

3.3 Update the position of the ant (Eq. 9).

Step 4 Compute the fitness of all ants and update matrix 1. The fitness is inversely proportional to the FS for the selected control variables and is calculated using Spencer's method explained in Sect. 3. The FS is computed only for matrices of ants $\left(M t_{O A n t}\right)$ and not for antlions when the computation is carried out inside the loop (refer to the flowchart in Fig. 5). Moreover, the elite antlion is always in updated antlion population, which is consistent with other evolutionary techniques that retain the optimal solution. 


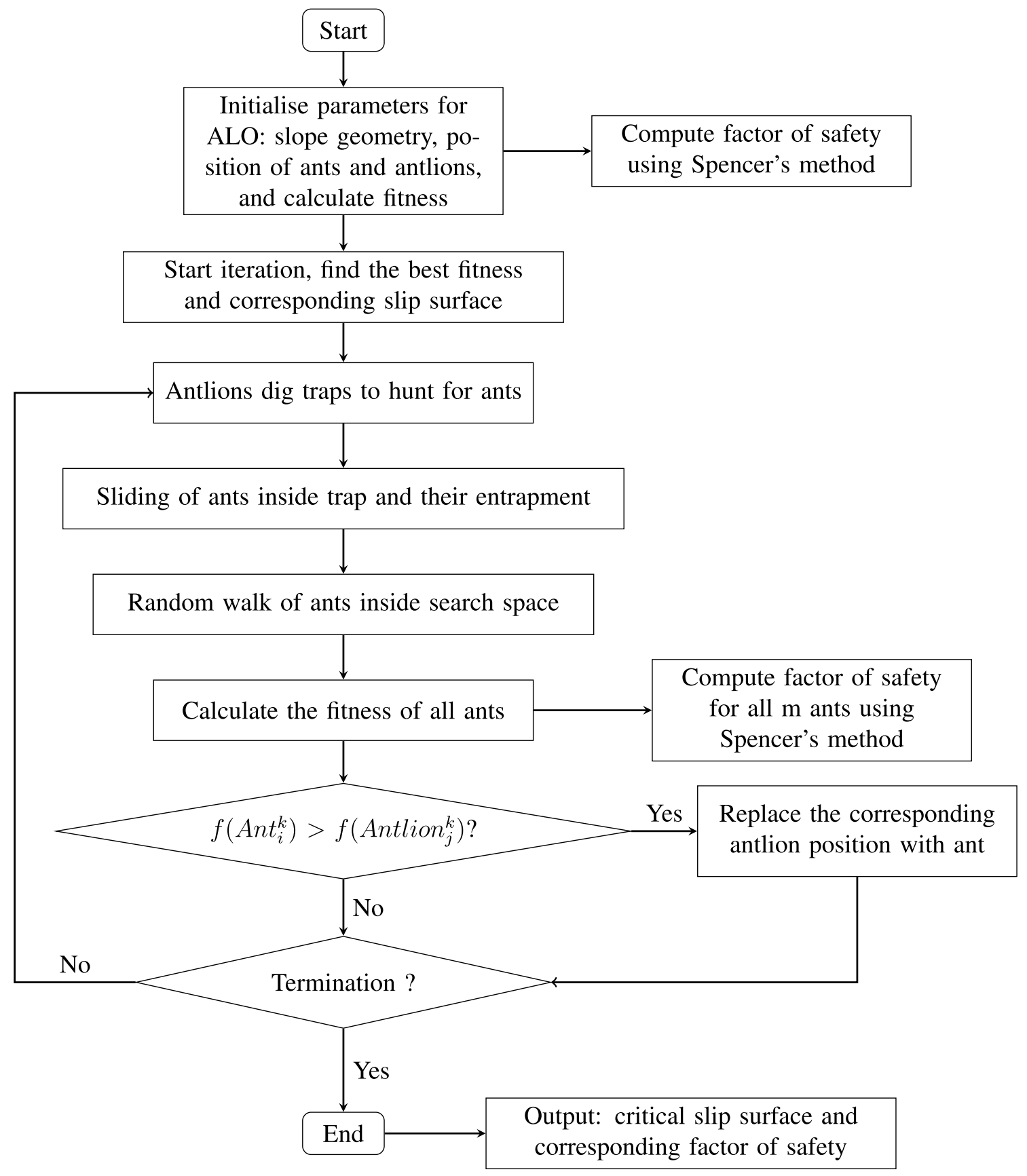

Fig. 5 Flowchart to capture critical slip surface and factor of safety using ALO algorithm for assessing slope stability

Step 5 The antlion exchanges its position with the corresponding ant if the ant is fitter. In this step, the fitness of the selected elite antlion (Eq. 10) improve, thus converging to the optimal solution.

Step 6 Replace elite if $\left(\right.$ fit Ant $_{i}$ (Iter $\left.)\right)<$ fit $\left(\right.$ Antlion $_{j}$ (Iter $\left.)\right)$.

Step 7 Repeat steps 3-6 until the termination criteria is reached.
The ALO solution comprises numerous generations of the system of variables that are optimised, resulting in the lowest FS. The position of the elitist antlion represents the critical slip surface with a minimum FS. A large value of fitness function is used when the FS violates the search boundary and generates infeasible slip surfaces. Figure 5 the process of the ALO algorithm adopted for slope stability. 
Table 2 Upper and lower limits of search variables and number of slices considered for examples $1-4$

\begin{tabular}{lccccccc}
\hline Example no. & Min & Max & Min & Max & Min & Max & No. of Slices \\
& $x_{I}$ & $x_{u}$ & $x_{L}$ & $x_{U}$ & $\sigma_{u}$ & $\sigma_{I}$ & N \\
\hline 1. & 0 & 10 & 20 & 30 & 0 & 1 & 30 \\
2. & 0 & 60 & 140 & 160 & 0 & 1 & 30 \\
3. & 10 & 15 & 25 & 34 & 0 & 1 & 30 \\
4. & 0 & 20 & 40 & 50 & 0 & 1 & 30 \\
\hline
\end{tabular}

Table 3 Control parameters used in antlion optimiser for factor of safety calculations in examples $1-4$

\begin{tabular}{lllll}
\hline Input variable sets & Example 1 & Example 2 & Example 3 & Example 4 \\
\hline $\begin{array}{l}\text { Population (Total number of ants and } \\
\text { antlions) (NP) }\end{array}$ & $10-50$ & $10-100$ & $10-100$ & $10-100$ \\
Maximum iterations count $\left(\right.$ Iter $_{\text {max }}$ ) & 50 & 100 & 100 & 100 \\
No. of control variables (Dim) & 31 & 31 & 31 & 31 \\
\hline
\end{tabular}

\section{Numerical experiments}

The section analyses four slopes with different complexities, namely a homogeneous slope and three heterogeneous slopes to test the efficacy of ALO approach. The critical slip surface and corresponding FS are determined through the proposed technique and compared with those obtained using other optimisation algorithms. Because the solution obtained in each simulation is different, 10 simulations are used for each numerical experiment to report the mean and standard deviation along with the optimal solution of the numerical experiment. Therefore, for 10 simulations for each numerical experiment, 10 optimal solutions are available for 10 experiments. Among these 10 optimal solution, a solution with the minimum FS is reported as the optimal solution. Moreover, Table 2 reports lower and upper bound limits of control variables. The total vertical slices are fixed to 30 because the computation of the FS of a slope is unaffected by the number of selected slices if the minimum number of slices is set to 30 [87]. Table 3 reports the parameters of ALO. The parameters of the ALO algorithm were chosen from previous studies [78]. Subsequent sections indicate that the critical slip surface gradually approached the optimal slip surface with the progression of the search for these two population sizes.

\subsection{Example 1}

The homogeneous soil slope considered from Yamagami and Ueta [88] is analysed. The slope is in dry condition with soil parameters reported in Table 4. Figure 6 presents slope geometry. Figure 6 analyses the critical slip surface identified by solving the same benchmark example by using other slope stability optimisaton methods
Table 4 Soil parameters for benchmark examples 1-4

\begin{tabular}{lllll}
\hline Example no. & Layer $(\mathrm{s})$ & $\begin{array}{l}\text { Cohesion (c) } \\
(\mathrm{kPa})\end{array}$ & $\begin{array}{l}\text { Friction } \\
\text { angle } \phi \\
\left({ }^{\circ}\right)\end{array}$ & Unit weight $\gamma$ \\
\hline 1. & 1 & 9.8 & 10 & $\mathrm{kN} / \mathrm{m}^{3}$ \\
\hline 2. & 1 & 28.7 & 20 & 17.64 \\
& 2 & 0 & 10 & 18.8 \\
3. & 1 & 15 & 20 & 19.0 \\
& 2 & 17 & 21 & 19.0 \\
& 3 & 5 & 10 & 19.0 \\
& 4 & 35 & 28 & 19.0 \\
4. & 1 & 0 & 38 & 19.5 \\
& 2 & 5.3 & 23 & 19.5 \\
& 3 & 7.2 & 20 & 19.5 \\
\hline
\end{tabular}

and the current technique. In the 10 computations, the calculated minimum and maximum FS are 1.317 and 1.349 , respectively. Table 5 presents a comparative summary for two population sizes used with its results verified against different methods. For the homogeneous case, irrespective of the swarm intelligence technique, most of them converge to similar results. The standard deviation in FS is reduced to 0.008 and 0.016 for populations of 50 and 10 from 0.298 , respectively, following the results provided by Kahatadeniya et al. [21] by using discrete ant colony optimisation. The current algorithm captures the optimal solution with a fewer number of adjustable parameters to adjust than several prominent algorithms. For the homogeneous case, the analysed algorithms converge to rather similar values, indicating the robustness of calculation.

Figure 7 presents the convergence behaviour of the ALO algorithm. Two simulations are plotted to indicate 
Fig. 6 Critical slip surface identified using ALO and other optimisation methods for example 1

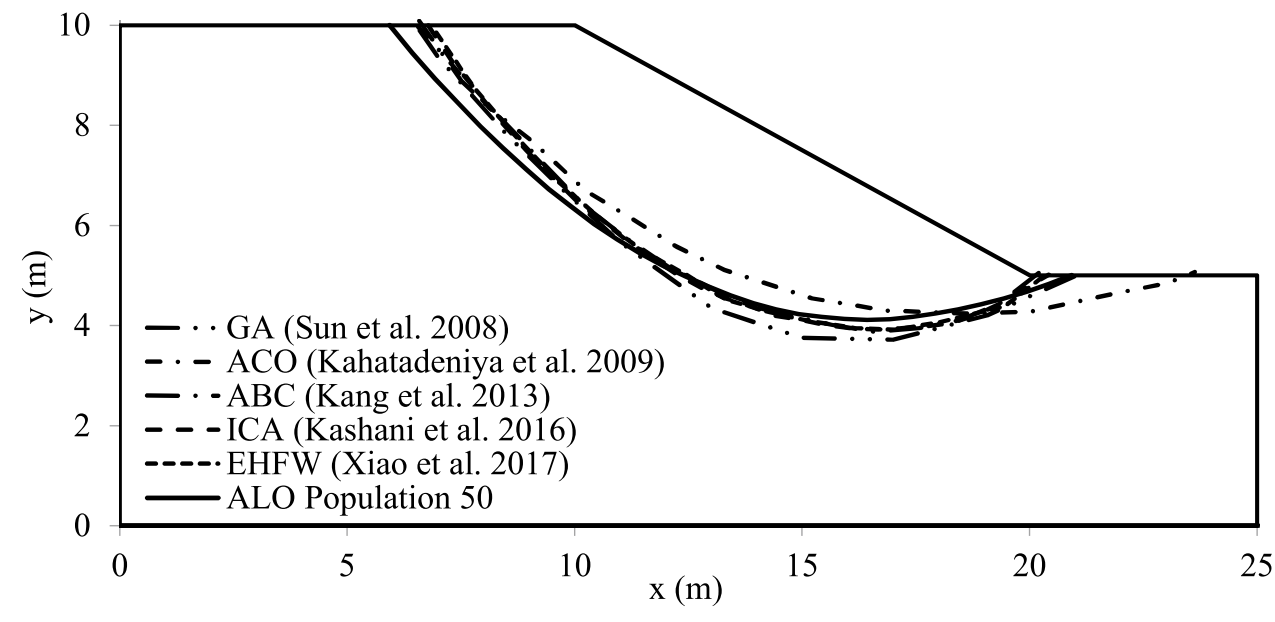

Table 5 Comparison of factor of safety obtained using ALO technique for the standard example 1 with different methods

\begin{tabular}{lll}
\hline Source & Method & FS \\
\hline Yamagami and Ueta [88] & Broyden-Fletcher-Goldfarb-Shanno (BFGS) & 1.338 \\
Yamagami and Ueta [88] & Simplex method & $1.339-1.348$ \\
Cheng et al. [87] & Particle swarm optimisation (PSO) & 1.329 \\
Cheng et al. [87] & Modified particle swarm optimisation (MPSO) & 1.326 \\
Cheng et al. [20] & Modified harmony search (MHS) & 1.322 \\
Jianping et al. [89] & Genetic algorithm (GA) + line & 1.324 \\
Jianping et al. [89] & Genetic algorithm (GA) + Spline & 1.321 \\
Kahatadeniya et al. [21] & Ant colony optimisation (ACO) & $1.311-2.966$ \\
Khajehzadeh et al. [25] & Particle swarm optimisation (PSO) & 1.321 \\
Khajehzadeh et al. [25] & Modified particle swarm optimisation (MPSO) & 1.308 \\
Kang et al. [27] & Artificial bee colony optimisation (ABC) \\
Kashani et al. [48] & Imperialistic competitive algorithm (ICA) & 1.321 \\
Xiao et al. [43] & Enhanced fireworks algorithm (EFW) & 1.321 \\
RS slope [90] & Cuckoo search & 1.322 \\
Mishra et al. [50] & Teaching-learning-based optimisation (TLBO) & 1.327 \\
ALO (This study) & Antlion Optimiser (ALO) NP = 10 & $1.324-1.325$ \\
ALO (This study) & Antlion Optimiser (ALO) NP = 50 & $1.334-1.387$ \\
\hline
\end{tabular}

that the fitness of the elitist antlion is updated using the number of iterations and converges to a constant value. The algorithm estimates the FS within desired accuracy,

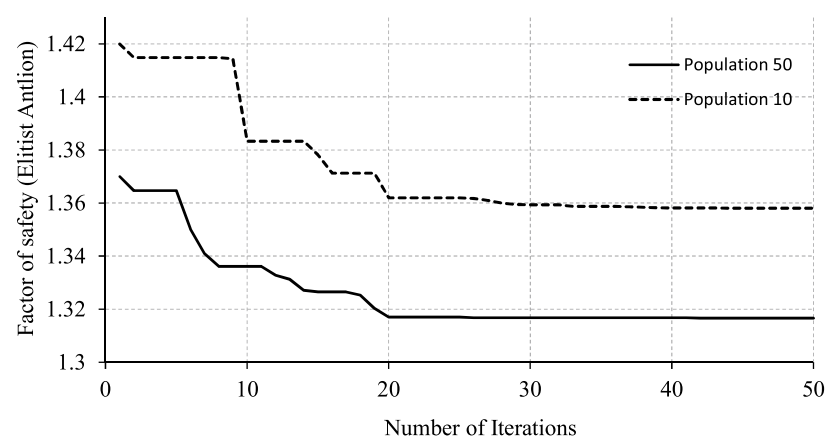

Fig. 7 Factor of safety for fittest antlion versus number of iterations by using populations of 50 and 10 for example 1 even with 10 ants and antlions. The initial estimate is already near to the optimal value and the difference between the optimal values obtained using the populations of 10 and 50 is residual -0.04 , which is negligible for engineering applications.

\subsection{Example 2}

The second illustrative example of the heterogeneous soil profile is referred to from Fredlund and Krahn [91]. Figure 8 presents the geometry of the slope, and soil parameters for three horizontal layers are reported in Table 4. Because of the presence of a thin band of a weak soil layer sandwiched between two strong soil layers, local minima occurs and several traditional methods fail to converge to global minima.

\section{SN Applied Sciences}




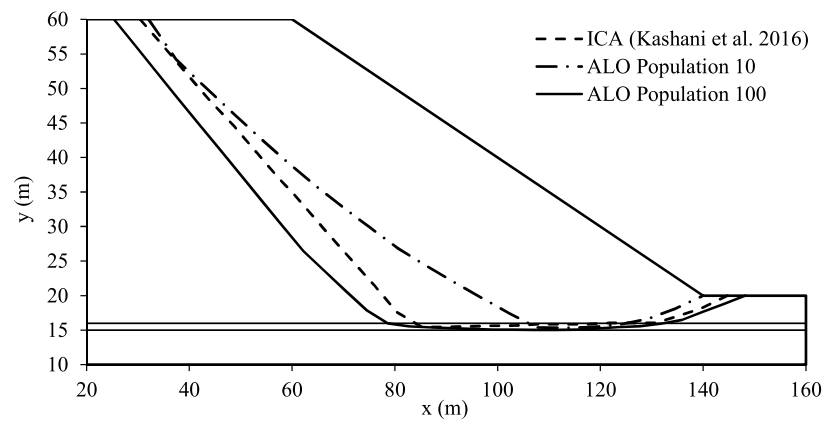

Fig. 8 Critical slip surface identified using ALO and ICA for example

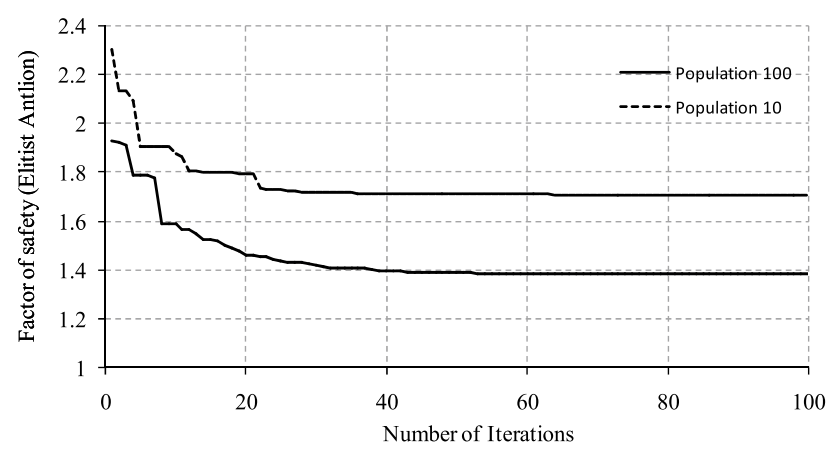

Fig. 9 Factor of safety for fittest antlion vs number of iterations using populations of 100 and 10 for example 2

Figure 8 presents the slip surface for populations of 10 and 100 identified using the current technique. It converges to the optimal solution by increasing the population of both ants and antlions to 100 . The current technique can automatically capture the critical failure surface. The obtained minimum FS is 1.366 , with a standard deviation of 0.091 . The method exhibits consistent results with less standard deviation and a superior estimation of the minimum factor of safety from Zhu et al. [94] and Jongmin et al. [93]. Figure 9 compares the convergence rates of the current technique for population sizes of 100 and 10 with 100 iterations. The population of ant and antlions for the heterogeneous layer must be approximately 100 to correctly identify global minima. The proposed technique is similar to the recent solution using ICA presented by Kashani et al. [48] and TLBO presented by Mishra et al. [50] with some minor deviations (refer Table 6), which may occur because of the discretisation of the sliding mass into slices and the method used for FS calculations.

\subsection{Example 3}

The third illustrative example of the heterogeneous soil profile having a weak band of an inclined soil layer is referred to from Zolfaghari et al. [95]. The soil parameters are reported in Table 4. In this case, the weak soil layer is sandwiched between two soil layers. Some optimisation techniques cannot converge to global minima.

The FS and corresponding slip surface have been computed using different optimisation techniques, such as genetic algorithm by Zolfaghari et al. [95]; tabu search, harmony search, particle swarm optimisation, simulated annealing, and ant colony optimisation by Cheng et al. [87]; gravitational search algorithm by Khajehzadeh et al. [30]; artificial bee colony by Kang et al. [27]; cuckoo search, firefly algorithm, and cuckoo search-boundary constraint (CS-BC) by Gandomi et al. [28]; imperialistic competitive algorithm by [48], as presented in Table 7 . The solution obtained using the proposed technique is 1.079 , which is the optimal solution for this problem. The solution is superior than those obtained using GA, SA, TS, ACO, HS, MHS, PSO, DE, ES FA and MVO. The results are a slightly on higher side when compared with CS, CS-BC, BBO and ICA, reason can be due to the method used to calculate $\mathrm{FS}$ as the difference is almost negligible. The standard deviation is obtained with ALO (0.162) is smaller than the one obtained by BBO (0.384) [46] but slightly more than MVO (0.1229) [49].

Figure 10 reports the critical slip surface obtained by solving the benchmark example by using the current
Table 6 Comparison of factor of safety obtained using ALO technique for the standard example 2 with different methods

\begin{tabular}{lll}
\hline Source & Method & FS \\
\hline Fredlund and Krahn [91] & Spencer's method & 1.373 \\
Goh [92] & Genetic algorithm & 1.288 \\
Jongmin et al. [93] & Limit equilibrium with the velocity field and plastic zone & 1.37 \\
Zhu et al. [94] & A generalised framework of LEM & 1.373 \\
Kashani et al. [48] & Imperialistic Competitive Algorithm (ICA) & 1.3625 \\
Mishra et al. [50] & Teaching-learning-based optimisation (TLBO) & $1.315-1.466$ \\
ALO (This study) & Antlion Optimiser (ALO) NP = 10 & $1.589-2.063$ \\
ALO (This study) & Antlion Optimiser (ALO) NP = 100 & $1.366-1.724$ \\
\hline
\end{tabular}


Table 7 Comparison of factor of safety obtained using ALO technique for the standard example 3 with different methods

Fig. 10 Critical slip surface identified using ALO and other optimisation methods for example 3

Fig. 11 Factor of safety for fittest antlion vs number of iterations using populations of 100 and 10 for example 4

\begin{tabular}{lll}
\hline Source & Method & FS \\
\hline Zolfaghari et al. [95] & Genetic algorithm (GA) & 1.24 \\
Cheng et al. [26] & Simulated annealing (SA) & 1.2813 \\
Cheng et al. [87] & Tabu search (TS) & 1.4661 \\
Cheng et al. [87] & Ant colony optimisation (ACO) & 1.5817 \\
Cheng et al. [87] & Harmony search (HS) & 1.2405 \\
Cheng et al. [87] & Modified harmony search (MHS) & 1.1315 \\
Cheng et al. [24] & Modified Particle swarm optimisation (MPSO) & 1.1289 \\
Cheng et al. [20] & Particle swarm optimisation (PSO) & 1.1095 \\
Khajehzadeh et al. [30] & Gravitational search algorithm & 1.0785 \\
Kang et al. [27] & Artificial bee colony optimisation (ABC) & 1.086 \\
Gandomi et al. [28] & Firefly algorithm (FA) & 1.303 \\
Gandomi et al. [28] & Cuckoo search (CS) & 1.0635 \\
Gandomi et al. [28] & Cuckoo search-boundary constraint (CS-BC) \\
Gandomi et al. [46] & Biogeography-based optimization (BBO) & 1.0502 \\
Gandomi et al. [46] & Differential evolution (DE) & 1.055 \\
Gandomi et al. [46] & Evolutionary strategy (ES) & 1.659 \\
Mishra et al. [49] & Multi verse optimiser (MVO) & 1.502 \\
ALO (This study) & Antlion optimiser (ALO) NP = 10 & $1.1447-1.7362$ \\
ALO (This study) & Antlion optimiser (ALO) NP = 100 & $1.253-1.906$ \\
\hline
\end{tabular}
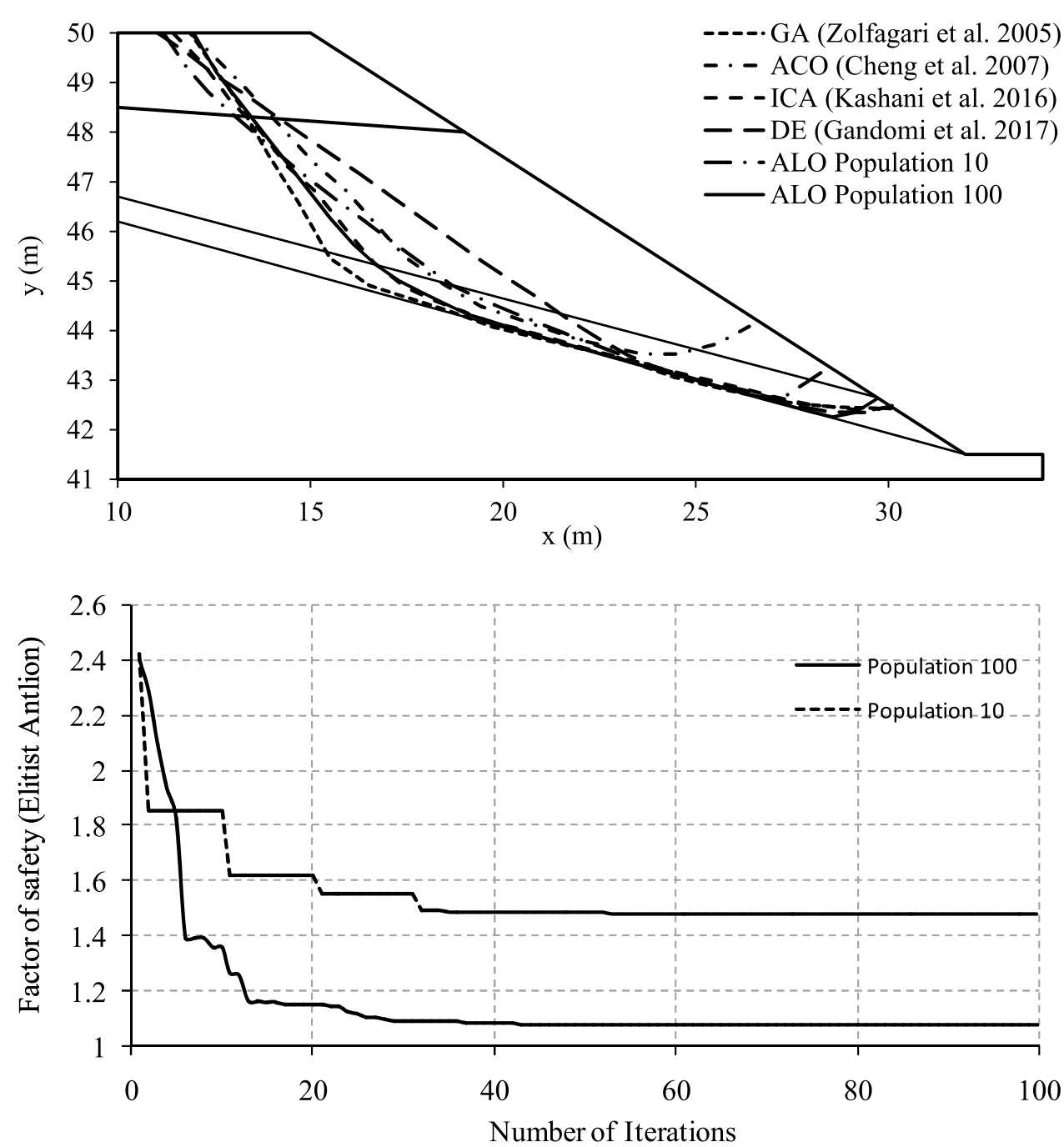
Fig. 12 Critical slip surface identified using ALO and other optimisation methods for example 3

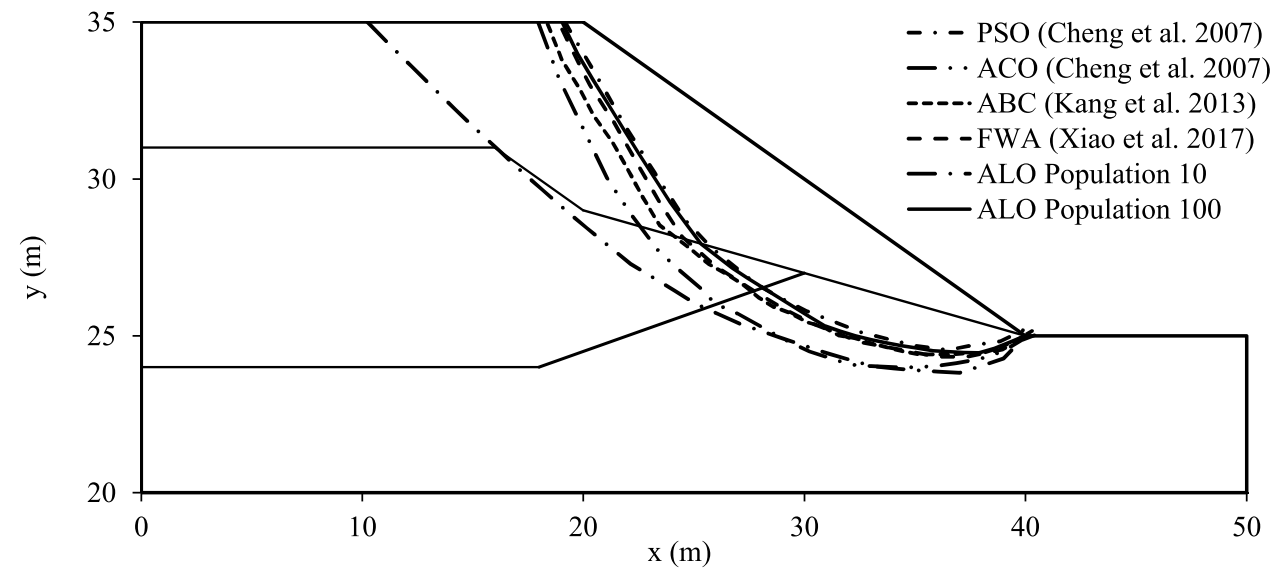

technique for the population sizes of 10 and 100 with 100 iterations. Figure 11 compares the convergence rate of ALO optimiser for population sizes of 10 and 100. For a population size of 100 , the ALO more rapidly converges to the optimal solution than with population of 10 .

\subsection{Example 4}

The fourth illustrative example of the heterogeneous soil profile is referred to from the Association for Computer Aided Designs (ACAD) in Australia [96]. The slope has three non-horizontal soil layers whose properties are reported in
Table 4. Figure 12 presents the slip surface for this example captured using the proposed technique. The critical surface obtained using the proposed algorithm is different for population sizes of 10 and 100 . Therefore, although the algorithm for both populations converges to similar FS values, the solution is different for each case. Both solutions present similar FS with the different geometry of the critical surface. The problem was previously analysed using $G A$, leap frog, $H S, S A, T S, H S, A B C, A C O, P P A C O, T L B O$ and finite element methods. The minimum factor of safety obtained using ALO is 1.351 with a standard deviation of 0.018 , indicating that this method is more efficient than
Table 8 Comparison of factor of safety obtained using ALO technique for the standard example 4 with different methods

\begin{tabular}{lll}
\hline Source & Method & FS \\
\hline Goh [92] & Genetic algorithm (GA) & 1.387 \\
Bolton et al. [97] & Leap frog method & 1.387 \\
Cheng et al. [87] & Particle swarm optimisation (PSO) & 1.359 \\
Cheng et al. [87] & Ant colony optimisation (ACO) & 1.3931 \\
Cheng et al. [87] & Simulated annealing (SA) & 1.3569 \\
Cheng et al. [87] & Tabu search (TS) & 1.3762 \\
Cheng el al. [20] & Harmony search algorithm (HSA) & 1.359 \\
Kang et al. [27] & Artificial bee colony optimisation (ABC) & 1.343 \\
Gao [45] & Evolutionary programming (EP) & 1.358 \\
Gao [45] & Immunised evolutionary programming (IEP) & 1.355 \\
Gao [22] & Ant colony optimisation (ACO) & 1.353 \\
Gao [23] & Meeting ant colony optimisation (MACO) & 1.348 \\
Gao [22] & Premium penalty ant colony optimisation (PPACO) \\
Goh [22] & Finite element method & 1.340 \\
Gao [98] & Improved black hole algorithm & 1.426 \\
Xiao et al. [43] & Enhanced fireworks algorithm (EFW) & 1.355 \\
Mishra et al. [50] & Teaching-learning-based optimisation (TLBO) & 1.350 \\
Mishra et al. [49] & Multi verse optimiser (MVO) & $1.343-1.356$ \\
ALO (This study) & Antlion optimiser (ALO) NP = 10 & $1.346-1.376$ \\
ALO (This study) & Antlion optimiser (ALO) NP = 100 & $1.571-1.714$ \\
\hline
\end{tabular}




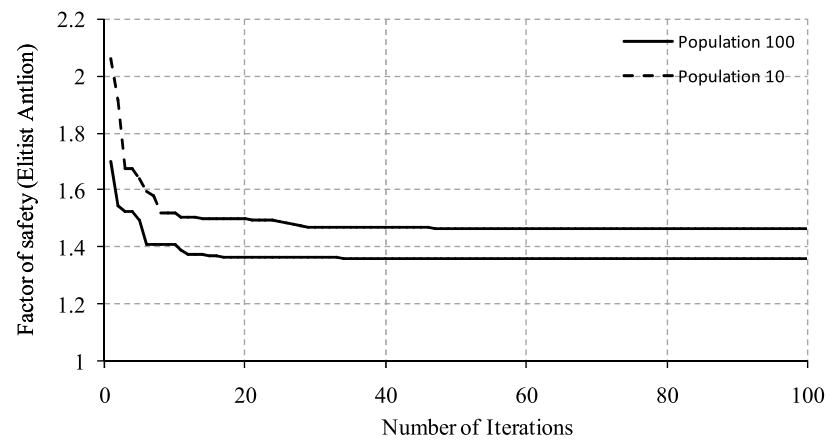

Fig. 13 Factor of safety for fittest antlion versus number of iterations using populations of 100 and 10 for example 4

most methods. However, TLBO presented by Mishra et al. [50] reports the FS between 1.343-1.356 with a standard deviation of 0.003193 making it more efficient in uncertainty reduction.

Table 8 reports the comparison of the FS obtained using the ALO technique for the standard example 4 with different methods previously reported. In this case, the result with FEM (1.426) is sub-optimal solution than result obtained by using the current method (1.351-1.404), which shows that current technique is robust. The ALO is more efficient than other studies because the FS values are similar. Figure 13 compares the convergence rates of ALO optimiser for population sizes of 10 and 100 with 100 iterations. For a population size of 100 , ALO more rapidly converges to optimal solution.

Finally, for comparison with other metaheuristic techniques, the number of fitness function evaluations described by NOFs is calculated under the same or replicated experimental conditions. Therefore, comparison is performed by calculating the total fitness evaluations for different population sizes and the number of iterations required for one simulation experiment. All the examples, excluding example 1 that has 2500 fitness function evaluations, used 10,000 fitness function evaluations. Figure 14 shows that the ALO algorithm has fewer fitness function evaluations than GA, PSO, and ACO for all four slopes and similar NOFs than $A B C$. Although $A B C$ and $A L O$ have the same number of fitness function evaluations, ALO has fewer function fitness function evaluations than GA, PSO, and ACO. In particular, for homogeneous slopes, the performance of ALO is superior in terms of accuracy and NOFs consumed (refer to a small bump shown in Fig. 14 for example 1 reporting 2500 NOFs) and for heterogeneous slopes it can locate the critical slip surface with success consuming 10,000 NOFs. The proposed approach yields accurate results when maintaining the number of fitness function evaluations at minimum in comparison with other optimisation techniques.

The computational time using population of 10 and 100 is further assessed based on a Intel(R) Core(TM) i5-7500 CPU $3.40 \mathrm{GHz}$ processors with $4.00 \mathrm{~GB}$ of installed memory (RAM). The average computing time is about $5 \mathrm{~s}$ for the population of 10 and $30 \mathrm{~s}$ for the population of 100 with 100 iterations for homogeneous slope. For heterogeneous slope profiles, the time increases to $7 \mathrm{~s}$ and $40 \mathrm{~s}$ for 100 iterations respectively.

\section{Conclusions}

A comprehensive state-of-the-art review of the slope stability using swarm intelligence techniques is presented in this paper. Additionally, from the state-of-theart reviews that the applications of swarm intelligence
Fig. 14 Comparison of total number of function evaluations (NOFs) of antlion optimisation with other optimisation techniques

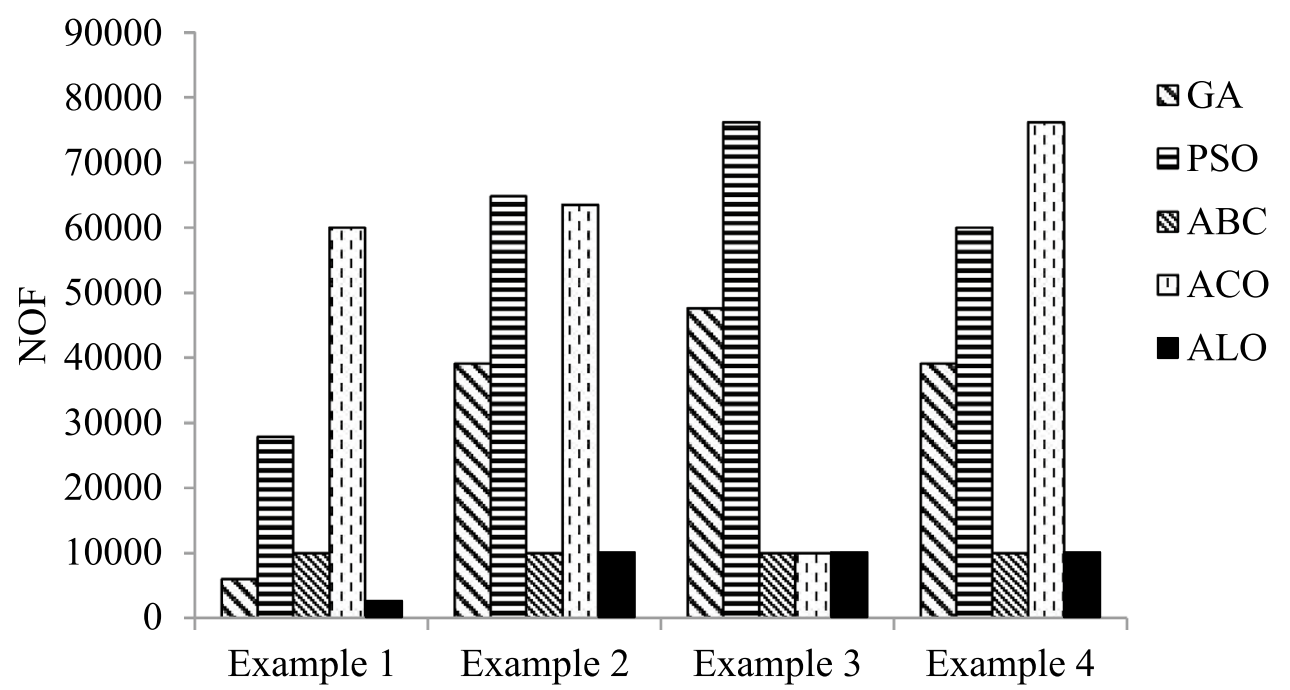


techniques are discussed with emphasis on parameter tuning, which is drawback of several methods. For the comparison purposes, the ALO algorithm is successfully adapted to solve the slope stability problem for four different examples with different complexities. On the basis of the four case studies analysed, the algorithm can reach satisfactory results, and therefore, it is robust and in line with other evolutionary algorithms already applied to the same cases in the past.

The fitness function evaluations for ALO is less than or similar to other optimisation approaches. It has been observed from the present study that the fitness function zeroed in on the global minima and unlike some bioinspired algorithms, it requires fewer parameters to adjust. One of the primary advantages of ALO over other prominent algorithms is that it has a fairly simple mathematical structure and requires only two parameters, namely the number of search agents and iterations, to be adjusted. ALO used roulette wheel selection and elite antlion bias to select control parameter as compared with ACO, which is primarily driven by pheromone level updates. Therefore, compared with ACO, the proposed method has wider search capabilities and can explore solutions by using more combinations of control variables. This mechanism assists ALO in coming out of local minimum more easily than its counterpart ACO. The results from numerical experiments demonstrate that solutions realised using ALO and TLBO have less standard deviation than those obtained using the existing optimisation methods. Therefore, the reviews of current study can be applied by various researchers and field practitioners for choosing a particular metaheuristic technique for their application.

The proposed analysis neglects the effect of pore water pressure as all the case study examples are analysed based on dry conditions (i.e. zero pore water pressure). Furthermore, stability evaluation of a slope in case of a seismic condition is not addressed in the paper. In the future works, effect of pore water pressure and earthquake forces can be taken into account as they have a direct effect on computing the safety factor of the slopes. Furthermore, effect of the number of vertical slices (current study 30 are used) on slopes of varying complexity and their effect on factor of safety can also be taken into consideration.

\section{Compliance with ethical standards}

Conflict of interest The authors declare that they have no conflict of interest.

\section{References}

1. Evans SG, Roberts NJ, Ischuk A, Delaney KB, Morozova GS, Tutubalina O (2009) Landslides triggered by the 1949 Khait earthquake, Tajikistan, and associated loss of life. Eng Geol 109(3-4):195-212

2. Ahmed B (2015) Landslide susceptibility modelling applying user-defined weighting and data-driven statistical techniques in Cox's Bazar Municipality, Bangladesh. Nat Hazards 79(3):707-1737

3. Ubydul H, Philipp B et al (2016) Fatal landslides in Europe. Landslides 13(6):1545-1554

4. Duric D, Mladenovic A, Pesic-Georgiadis M et al (2017) Using multiresolution and multitemporal satellite data for post-disaster landslide inventory in the Republic of Serbia. Landslides 14(4):1467-1482

5. Cheng YM, Lansivaara T, Wei WB (2007) Two-dimensional slope stability analysis by limit equilibrium and strength reduction methods. Comput Geotech 34(3):137-150

6. Bishop AW (1955) The use of the slip circle in the stability analysis of slopes. Géotechnique 5:7-17

7. Janbu N (1973) Slope stability computations. In: Hirschfeld RC, Poulos SJ (eds) Embankment dam engineering. Wiley, New York

8. Morgenstern NR, Price VE (1965) The analysis of the stability of general slip surfaces. Géotechnique 15(1):79-93

9. Viggiani C (1981) Ultimate lateral load on piles used to stabilize landslides. In: Proceedings of the 10th international conference on soil mechanics and foundation engineering, Balkema, Rotterdam, vol. 3, pp 555-560

10. Zhu DY, Lee CF (2002) Explicit limit equilibrium solution for slope stability. Int J Numer Anal Methods Geomech 26:1573-1590

11. Li X, Su L, He S, Xu J (2016) Limit equilibrium analysis of seismic stability of slopes reinforced with a row of piles. Int $J$ Numer Anal Methods Geomech 40:1241-1250

12. Chen ZY, Shao CM (1988) Evaluation of minimum factor of safety in slope stability analysis. Can Geotech J 25(4):735-748

13. Fast Lagrangian Analysis of Continua (FLAC) (2005) version 5.0. ITASCA Consulting Group, Inc., Minneapolis

14. Duncan JM (1996) State of the art: limit equilibrium and finiteelement analysis of slopes. J Geotech Eng 122(7):577-596

15. Griffiths DV, Lane PA (1999) Slope stability analysis by finite elements. Géotechnique 49(3):387-403

16. Zheng H, Sun G, Liu D (2009) A practical procedure for searching critical slip surfaces of slopes based on the strength reduction technique. Comput Geotech 36(1):1-5

17. Nasvi MCM, Krishnya S (2019) Stability analysis of ColomboKatunayake expressway (CKE) using finite element and limit equilibrium methods. Indian Geotech J. https://doi. org/10.1007/s40098-019-00357-7

18. Mahdiyar A, Hasanipanah M, Armaghani DJ et al (2017) A Monte Carlo technique in safety assessment of slope under seismic condition. Eng Comput 33(4):807-817

19. Mojtahedi SFF, Tabatabaee S, Ghoroqi M et al (2018) A novel probabilistic simulation approach for forecasting the safety factor of slopes: a case study. Eng Comput 35:1-10

20. Cheng YM, Li L, Lansivaara T, Chi SC, Sun YJ (2008) An improved harmony search minimization algorithm using different slip surface generation methods for slope stability analysis. Eng Optim 40(2):95-115

21. Kahatadeniya KS, Nanakorn P, Neaupane KM (2009) Determination of the critical failure surface for slope stability analysisusing ant colony optimization. Eng Geol 108:133-141 
22. Gao W (2016) Premium-penalty ant colony optimization and its application in slope stability analysis. Appl Soft Comput 43:480-488

23. Gao W (2016) Determination of non-circular critical slip surface in slope stability analysis by meeting ant colony optimization. J Comput Civil Eng ASCE 30(2):06015001

24. Cheng YM, Li L, Chi S, Wei WB (2007) Particle swarm optimization algorithm for the location of the critical non-circular failure surface in two-dimensional slope stability analysis. Comput Geotech 34(2):92-103

25. Khajehzadeh M, Taha MR, El-Shafie A, Eslami M (2012) Locating the general failure surface of earth slope using particle swarm optimization. Civil Eng Environ Syst 29(1):41-57

26. Cheng YM (2007) Global optimization analysis of slope stability by simulated annealing with dynamic bounds and dirac function. Eng Optim 39(1):17-32

27. Kang F, Li J, Ma Z (2013) An artificial bee colony algorithm for locating the critical slip surface in slope stability analysis. Eng Optim 45(2):207-223

28. Gandomi AH, Kashani AR, Mousavi M, Jalalvandi M (2014) Slope stability analyzing using recent swarm intelligence techniques. Int J Numer Anal Methods Geomech 39(3):295-309

29. Cheng YM, Liang L, Chi SC, Wei WB (2008) Determination of the critical slip surface using artificial fish swarms algorithm. J Geotech Geoenviron Eng 134(2):244-251

30. Khajehzadeh M, Taha MR, El-shafie A, Eslami M (2011) Search for critical failure surface in slope stability analysis by gravitational search algorithm. Int J Phys Sci 6(21):5012-5021

31. Khajehzadeh M, Taha MR, El-Shafie A, Eslami M (2012) A modified gravitational search algorithm for slope stability analysis. Eng Appl Artif Intell 25(8):1589-1597

32. Singh J, Banka H, Verma AK (2018) Analysis of slope stability and detection of critical failure surface using gravitational search algorithm. In: 4th International conference on recent advances in information technology (RAIT), Dhanbad, pp 1-6

33. Saha A (2013) Big-bang big-crunch optimization in locating the critical surface in slope-stability. In: Proceedings of Indian geotechnical conference, Roorkee, Paper No. 82

34. Zhao H, Yin S, Ru Z (2012) Relevance vector machine applied to slope stability analysis. Int J Numer Anal Methods Geomech 36(5):643-652

35. Hu C, Jimenez R, Li SC, Li LP (2013) Determination of critical slip surfaces using mutative scale chaos optimization. J Comput Civil Eng 29(5):04014067

36. Tongchun H (2012) Stability calculation of slope by a tabu search method. In: Fourth joint international symposium on information technology in civil engineering, towards a vision for information technology in civil engineering

37. Sengupta A, Upadhyay A (2009) Locating the critical failure surface in a slope stability analysis by genetic algorithm. Appl Soft Comput 9(1):387-392

38. Pina RJ, Jimenez R (2015) A genetic algorithm for slope stability analyses with concave slip surfaces using custom operators. Eng Optim 47(4):453-472

39. Manouchehrian A, Gholamnejad J, Sharifzadeh M (2014) Development of a model for analysis of slope stability for circular mode failure using genetic algorithm. Environ Earth Sci 71(3):1267-1277

40. Zhu JF, Chen CJ (2014) Search for circular and noncircular critical slip surfaces in slope stability analysis by hybrid genetic algorithm. J Central South Univ 21(1):387-397

41. McCombie P, Wilkinson $P$ (2002) The use of the simple genetic algorithm in finding the critical factor of safety in slope stability analysis. Comput Geotech 29(8):699-714

42. Goh ATC (2000) Search for critical slip circle using genetic algorithms. Civil Eng Environ Syst 17(3):181-211
43. Xiao Z, Tian B, Lu X (2017) Locating the critical slip surface in a slope stability analysis by enhanced fireworks algorithm. Cluster Comput 22:1-11

44. Gao W, Wang X, Dai S, Chen D (2016) Study on stability of high embankment slope based on black hole algorithm. Environ Earth Sci 75(20):1381

45. Gao W (2015) Slope stability analysis based on immunised evolutionary programming. Environ Earth Sci 74(4):3357-3369

46. Gandomi AH, Kashani AR, Mousavi M, Jalalvandi M (2017) Slope stability analysis using evolutionary optimization techniques. Int J Numer Anal Methods Geomech 41(2):251-264

47. Singh J, Banka H, Verma AK (2018) A BBO-based algorithm for slope stability analysis by locating critical failure surface. Neural Comput Appl 31:1-18

48. Kashani AR, Gandomi AH, Mousavi M (2016) Imperialistic competitive algorithm: a metaheuristic algorithm for locating the critical slip surface in 2-dimensional soil slopes. Geosci Front 7(1):83-89

49. Mishra M, Gunturi RV, Maity D (2019a) Multiverse optimisation algorithm for capturing the critical slip surface in slope stability analysis. Geotech Geol Eng. https://doi.org/10.1007/s1070 6-019-01037-2

50. Mishra M, Gunturi RV, Maity D (2019b) Teaching-learning-based optimisation algorithm and its application in capturing critical slip surface in slope stability analysis. Soft Comput. https://doi. org/10.1007/s00500-019-04075-3

51. Gandomi AH, Kashani AR, Mousavi M (2015) Boundary constraint handling affection on slope stability analysis. In: Lagaros N, Papadrakakis $M$ (eds) Engineering and applied sciences optimization. Springer, Basel, pp 341-358

52. Liang L, Xue-Song C (2011) An improved particle swarm optimization algorithm with harmony strategy for the location of critical slip surface of slopes. China Ocean Eng 25(2):357-364

53. Dong H, Jian-Ping Q (2010) Hybrid of ant colony algorithm and simulated annealing algorithm and its application to the slope stability analysis. In: 2010 Sixth international conference on natural computation vol. 6, pp 3329-3333

54. Raihan TM, Mohammad K, Mahdiyeh E (2011) A new hybrid algorithm for global optimization and slope stability evaluation. J Central South Univ 20(11):3265-3273

55. Sakellariou MG, Ferentinou MD (2005) A study of slope stability prediction using neural networks. Geotech Geol Eng 23(4):419-445

56. Kaunda RB, Chase RB, Kehew AE, Kaugars K, Selegean JP (2010) Neural network modeling applications in active slope stability problems. Environ Earth Sci 60(7):1545-1558

57. Choobbasti AJ, Farrokhzad F, Barari A (2009) Prediction of slope stability using artificial neural network (case study: Noabad, Mazandaran, Iran). Arab J Geosci 2(4):311-319

58. Erzin Y, Cetin T (2012) The use of neural networks for the prediction of the critical factor of safety of an artificial slope subjected to earthquake forces. Scientia Iranica 19(2):188-194

59. Chok YH, Jaksa MB, Kaggwa WS, Griffiths DV, Fenton GA (2016) Neural network prediction of the reliability of heterogeneous cohesive slopes. Int J Numer Anal Methods Geomech 40:1556-1569

60. Hoang N, Pham A (2016) Hybrid artificial intelligence approach based on metaheuristic and machine learning for slope stability assessment: a multinational data analysis. Expert Syst Appl 46:60-68

61. Kang F, Li J, Xu Q (2017) System reliability analysis of slopes using multilayer perceptron and radial basis function networks. Int J Numer Anal Methods Geomech 41:1962-1978

62. Das SK, Biswal RK, Sivakugan N, Das B (2011) Classification of slopes and prediction of factor of safety using differential evolution neural networks. Environ Earth Sci 64(1):201-210 
63. Mahdevari S, Shahriar K, Sharifzadeh M et al (2017) Stability prediction of gate roadways in longwall mining using artificial neural networks. Neural Comput Appl 28(11):3537-3555

64. Gordan B, Jahed-Armaghani D, Hajihassani M, Monjezi M (2016) Prediction of seismic slope stability through combination of particle swarm optimization and neural network. Eng Comput 32:85-97

65. Zhang RH, Goh ATC, Zhang WG (2019) System reliability assessment on deep braced excavation adjacent to an existing upper slope in mountainous terrain: a case study SN. Appl Sci 1:876

66. Ye S, Fang G, Ma X (2019) Reliability analysis of grillage flexible slope supporting structure with anchors considering fuzzy transitional interval and fuzzy randomness of soil parameters. Arab J Sci Eng 44(10):8849-8857. https://doi.org/10.1007/s1336 9-019-03912-9

67. Qi C, Tang X (2018) A hybrid ensemble method for improved prediction of slope stability. Int J Numer Anal Methods Geomech 42:1823-1839

68. Wolpert DH, Macready WG (1997) No free lunch theorems for optimization. IEEE Trans Evolut Comput 1(1):67-82

69. Bagheri Sereshki A, Derakhshani A (2019) Optimizing the mechanical stabilization of earth walls with metal strips: applications of swarm algorithms. Arab J Sci Eng 44(5):4653. https:// doi.org/10.1007/s13369-018-3492-8

70. Kaveh A, Behnam AF (2013) Charged system search algorithm for the optimum cost design of reinforced concrete cantilever retaining walls. Arab J Sci Eng 38(3):563-570. https://doi. org/10.1007/s13369-012-0332-0

71. Mirjalili S (2015) The ant lion optimizer. Adv Eng Softw 83:80-98

72. Ali ES, Abd-Elazim SM, Abdelaziz AY (2016) Ant lion optimization algorithm for renewable distributed generations. Energy 116(1):445-458

73. Zawbaa HM, Emary E, Parv B (2015) Feature selection based on antlion optimization algorithm. Third World Conf Complex Syst 2015:1-7

74. Kamboj VK, Bhadoria A, Bath SK (2017) Solution of non-convex economic load dispatch problem for small-scale power systems using ant lion optimizer. Neural Comput Appl 28(8):2181-2192

75. Kilic H, Yuzgec U, Karakuzu C (2018) A novel improved antlion optimizer algorithm and its comparative performance. Neural Comput Appl. https://doi.org/10.1007/s00521-018-3871-9

76. Mani M, Bozorg-Haddad O, Chu X (2018) Ant lion optimizer (ALO) algorithm. In: Bozorg-Haddad O (ed) Advanced optimization by nature-inspired algorithms. Studies in computational intelligence, vol 720. Springer, Singapore, pp 105-116. https:// doi.org/10.1007/978-981-10-5221-7_11

77. Kaveh M, Amiri Chayjan R, Taghinezhad E, Abbaspour Gilandeh Y, Younesi A, Rasooli Sharabiani V (2019) Modeling of thermodynamic properties of carrot product using ALO, GWO, and WOA algorithms under multi-stage semi-industrial continuous belt dryer. Eng Comput 35(3):1045-1058. https://doi.org/10.1007/ s00366-018-0650-2

78. Mishra M, Barman SK, Maity D, Maiti DK (2019c) Ant lion optimisation algorithm for structural damage detection using vibration data. J Civil Struct Health Monit 9(1):117-136. https://doi. org/10.1007/s13349-018-0318-z

79. Subhashini KR, Satapathy JK (2017) Development of an enhanced ant lion optimization algorithm and its application in antenna array synthesis. Appl Soft Comput 59:153-173
80. Crepinšek M, Liu SH, Mernik L, Mernik M (2016) Is a comparison of results? Meaningful from the inexact replications of computational experiments? Soft Comput 20(1):223-235

81. Mernik M, Liu SH, Karaboga D, Crepinšek M (2015) On clarifying misconceptions when comparing variants of the artificial bee colony algorithm by offering a new implementation. Inf Sci 291:115-127

82. MATLAB (2010) version 7.10.0 (R2010a). The MathWorks Inc., Natick, Massachusetts

83. Cheng YM (2003) Locations of critical failure surface and some further studies on slope stability analysis. Comput Geotech 30:255-267

84. Lo S-CR, Xu D-W (1992) A strain based design method for the collapse limit state of reinforced soil walls and slopes. Can Geotech J 29(8):832-842

85. Zhu DY, Lee CF, Qian QH, Zou ZS, Sun F (2005) A concise algorithm for computing the factor of safety using the MorgensternPrice method. Can Geotech J 42(1):272-278

86. Spencer E (1967) A method of analysis of the stability of embankments assuming parallel inter-slice forces. Géotechnique 17:11-26

87. Cheng YM, Li L, Chi SC (2007) Performance studies on six heuristic global optimization methods in the location of critical failure surface. Comput Geotech 34:462-484

88. Yamagami T, Ueta Y (1988) Search for noncircular slip surfaces by the morgenstern-price method. In: The sixth international conference numerical methods in geomechanics, pp 1335-1340

89. Jianping S, Li J, Liu Q (2008) Search for critical slip surface in slope stability analysis by spline-based GA method. J Geotech Geoenviron Eng 134(2):252-256

90. Rocscience (2002) Slide, a synopsis of slope stability analysis. Technical report, Rocscience

91. Fredlund DG, Krahn J (1977) Comparison of slope stability methods of analysis. Can Geotech J 13(3):429-439

92. Goh ATC (1999) Genetic algorithm search for critical slip surface in multiple-wedge stability analysis. Can Geotech J 36:382-391

93. Jongmin K, Salgado R, Juhnwan L (2002) Stability analysis of complex soil slopes using limit analysis. J Geotech Geoenviron Eng 7:546-557

94. Zhu DY, Lee CF, Jiang HD (2003) Generalised framework of limit equilibrium methods for slope stability analysis. Géotechnique 53(4):377-395

95. Zolfaghari AR, Heath AC, McCombie PF (2005) Simple genetic algorithm search for critical non-circular failure surface in slope stability analysis. Comput Geotech 32(3):139-152

96. Donald IB (1989) Soil slope stability programs review. Association for computer aided design, review (Australia)

97. Bolton HPJ, Heymann G, Groenwold AA (2003) Global search for critical failure surface in slope stability analysis. Eng Optim 35(1):51-65

98. Gao W (2017) Investigating the critical slip surface of soil slope based on an improved black hole algorithm. Soils Found 57:988-1001

Publisher's Note Springer Nature remains neutral with regard to jurisdictional claims in published maps and institutional affiliations. 